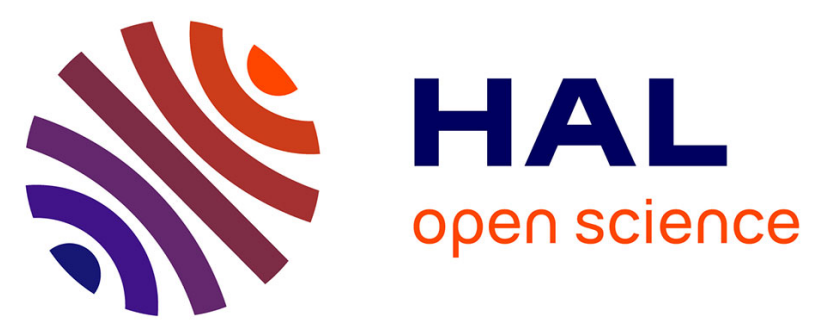

\title{
Level of PICALM, a key component of clathrin-mediated endocytosis, is correlated with levels of phosphotau and autophagy-related proteins and is associated with tau inclusions in AD, PSP and Pick disease
}

Kunie Ando, Karen Tomimura, Véronique Sazdovitch, Valérie Suain, Zehra Yilmaz, Michèle Authelet, Marième Ndjim, Cristina Vergara, Mounir Belkouch, Marie-Claude Potier, et al.

\section{- To cite this version:}

Kunie Ando, Karen Tomimura, Véronique Sazdovitch, Valérie Suain, Zehra Yilmaz, et al.. Level of PICALM, a key component of clathrin-mediated endocytosis, is correlated with levels of phosphotau and autophagy-related proteins and is associated with tau inclusions in AD, PSP and Pick disease. Neurobiology of Disease, 2016, 94, pp.32-43. 10.1016/j.nbd.2016.05.017 . hal-01332101

\section{HAL Id: hal-01332101 \\ https: / hal.sorbonne-universite.fr/hal-01332101}

Submitted on 15 Jun 2016

HAL is a multi-disciplinary open access archive for the deposit and dissemination of scientific research documents, whether they are published or not. The documents may come from teaching and research institutions in France or abroad, or from public or private research centers.
L'archive ouverte pluridisciplinaire HAL, est destinée au dépôt et à la diffusion de documents scientifiques de niveau recherche, publiés ou non, émanant des établissements d'enseignement et de recherche français ou étrangers, des laboratoires publics ou privés. 
Level of PICALM, a key component of clathrin-mediated endocytosis, is correlated with levels of phosphotau and autophagy-related proteins and is associated with tau inclusions in AD, PSP and Pick disease

Kunie Ando ${ }^{1,2,3}$, Karen Tomimura ${ }^{1,2}$, Véronique Sazdovitch ${ }^{1}$, Valérie Suain ${ }^{3}$, Zehra Yilmaz ${ }^{3}$, Michèle Authelet $^{3}$, Marième Ndjim², Cristina Vergara ${ }^{3}$, Mounir Belkouch ${ }^{1,2}$, Marie-Claude Potier $^{2}$, Charles Duyckaerts*1, ${ }^{1}$, Jean-Pierre Brion*3

${ }^{1}$ Laboratoire de Neuropathologie Escourolle, Hôpital de la Pitié-Salpêtrière, AP-HP, Paris, France

${ }^{2}$ Sorbonne Universités, UPMC Univ Paris 06 UMR S 1127, and Inserm, U 1127, and CNRS UMR 7225, and ICM, F-75013, Paris, France

${ }^{3}$ Laboratory of Histology, Neuroanatomy and Neuropathology, UNI (ULB Neuroscience Institute), Université Libre de Bruxelles, B-1070 Brussels, Belgium.

21 pages

6 figures

1 Supplementary figure

1 table

Abstract: 227 words

Running title: PICALM in tauopathies

*: authors equally contributed

\section{Corresponding authors:}

Dr. Jean-Pierre Brion

Laboratory of Histology, Neuroanatomy and Neuropathology, UNI (ULB Neuroscience Institute), Université Libre de Bruxelles,

808, route de Lennik (Bldg G)

B-1070 Brussels, Belgium.

Email: jpbrion@ulb.ac.be

Tel: +3225556505

Dr Charles Duyckaerts

Laboratoire de Neuropathologie Escourolle, Hôpital de la Pitié-Salpêtrière,

Boulevard de L'Hôpital, 47 - 83

75651 CEDEX 13 Paris

Paris, France

Email: charles.duyckaerts@aphp.fr

Tel: +33142161891 


\begin{abstract}
Single nucleotide polymorphisms in PICALM, a key component of clathrin-mediated endocytosis machinery, have been identified as genetic susceptibility loci for late onset Alzheimer's disease (LOAD). We previously reported that PICALM protein levels were decreased in AD brains and that PICALM was co-localised with neurofibrillary tangles in LOAD, familial AD with PSEN1 mutations and Down syndrome. In the present study, we analysed PICALM expression, cell localisation and association with pathological cellular inclusions in other tauopathies and in non-tau related neurodegenerative diseases. We observed that PICALM was associated with neuronal tau pathology in Pick disease and in progressive supranuclear palsy (PSP) and co-localised with both $3 \mathrm{R}$ and $4 \mathrm{R}$ tau positive inclusions unlike in corticobasal degeneration (CBD) or in frontotemporal lobar degeneration (FTLD)-MAPT P301L. PICALM immunoreactivities were not detected in tau-positive tufted astrocytes in PSP, astrocytic plaques in CBD, Lewy bodies in Lewy body disease, diffuse type (LBD) and in TDP-43-positive inclusions in FTLD. In the frontal cortex in tauopathies, the ratio of insoluble to soluble PICALM was increased while the level of soluble PICALM was decreased and was inversely correlated with the level of phosphotau. PICALM decrease was also significantly correlated with increased LC3-II and decreased Beclin-1 levels in tauopathies and in non-tau related neurodegenerative diseases. These results suggest that there is a close relationship between abnormal PICALM processing, tau pathology and impairment of autophagy in human neurodegenerative diseases.
\end{abstract}

Key words: PICALM, tau, NFTs, Alzheimer's disease, Pick disease, progressive supranuclear palsy, corticobasal degeneration, FTLD-MAPT, FTLD-TDP, diffuse Lewy body disease, autophagy, LC3, Beclin-1 


\section{Introduction}

Tauopathies are a group of disorders in which hyperphosphorylation and abnormal aggregation of the microtubule associated protein tau (MAPT) in brain tissue occur as a major pathological characteristic. In neurons, tau plays essential roles in intracellular cargo transport by polymerizing and stabilizing microtubules, but is also involved in other functions such as signal transduction, interaction with actin cytoskeleton, neuronal activity, synaptic plasticity and integrity of genomic DNA (Buee et al., 2000; Wang and Mandelkow, 2016). Tauopathies include Alzheimer disease (AD), the most common form of dementia, neuropathologically characterized by intraneuronal accumulation of highly phosphorylated tau as neurofibrillary tangles (NFTs), neuropil threads and extracellular deposition of A $\beta$ (Duyckaerts et al., 2009). Other tauopathies include frontotemporal lobar degeneration (FTLD) with tau inclusions, mainly due to mutations of the MAPT gene, Pick disease, progressive supranuclear palsy (PSP), and corticobasal degeneration (CBD) (Feany and Dickson, 1996; Mackenzie et al., 2009). The morphological appearance of tau lesions differs according to the disease. Some are highly specific such as Pick bodies in Pick disease, tufted astrocytes in PSP, astrocytic plaques in CBD. In FTLD-MAPT mutation at P301L brains, severe neuronal loss occurs in frontal and temporal cortex with the presence of gliosis and ballooned cells. Pre-tangles and perinuclear tau accumulation are characteristics of cases with FTLD-MAPT P301L (Spillantini et al., 1998). Pick bodies are made of three-repeat tau (3R-tau) and tau inclusions in PSP and CBD are made of four-repeat tau (4R-tau) while Alzheimer NFTs are composed of both 3R- and 4R-tau (Buee et al., 2000). The profile of affected brain regions is specific to each of these diseases (Buee et al., 2000; Lee et al., 2001; Dickson et al., 2007; Kovacs, 2015). Other neurodegenerative diseases are characterized by cellular inclusions composed of other proteins: e.g FTLD may be associated with TDP-43 positive inclusions or neurites (FTLD-TDP) (Neumann et al., 2006; Mackenzie et al., 2009); Lewy body disease, diffuse type (LBD) is associated to $\alpha$-synuclein positive inclusions (Spillantini et al., 1997). Molecular partners have been identified in some of these inclusions and might play a role in their formation and in the cellular mechanisms of the associated diseases.

Recent genome wide association studies (GWAS) have identified several new genetic risk factors for late onset Alzheimer's disease (LOAD). Two single nucleotide polymorphisms (SNPs) in the 5' region of PICALM gene have been reported as significant genetic risk loci for LOAD in several studies (Harold et al., 2009; Lambert et al., 2009; Seshadri et al., 2010; Naj et al., 2011; Lambert et al., 2013). Phosphatidylinositol binding clathrin assembly protein, PICALM (aka CALM) assembles adaptor protein-2 (AP-2) to clathrin, thus participating in clathrin-mediated endocytosis. We have previously reported that the level of full-length PICALM is decreased in AD brains; PICALM was co-localised with phosphorylated tau in NFTs and in granulovacuolar degenerations (GVDs) in the brains of AD patients and of individuals with Down syndrome but was not observed in amyloid plaques (Ando et al., 2013). Recently, PICALM was also found to modulate autophagy and to alter clearance of tau in cultured cells, in zebrafish and in Drosophila (Moreau et al., 2014). PICALM was also found to be expressed in endothelial cells in human brain tissue and could thus regulate $A B$ endothelial transcytosis and $A B$ clearance (Baig et al., 2010). Reduced expression of PICALM in murine brain endothelium accelerated Aß deposition in APP mice (Zhao et al., 2015). It remains however unclear whether PICALM-tau association is AD specific or could take place in other tauopathies or in diseases with tau-negative cellular inclusions. To extend our knowledge on the association of PICALM with tau pathology, we set out in this study to analyse the 
expression of PICALM and its localisation in the brain in several tauopathies or in neurodegenerative disorders without tau inclusions. 


\section{Material and methods}

\section{Antibodies}

Rabbit polyclonal anti-PICALM (HPA019053) and mouse monoclonal anti- $\beta$ actin (A5441) antibodies were purchased from SIGMA. Mouse monoclonal anti-pSer202/Thr205 tau (AT8) antibody was purchased from Pierce (MN1020). Mouse monoclonal anti-tau 3R isoform RD3 (05-803) and anti-tau 4R isoform RD4 (05-804) antibodies were purchased from Millipore. Mouse monoclonal pSer396/404 tau (PHF1) antibody was kindly provided by Dr. Peter Davies (Albert Einstein College of Medicine, NY). The rabbit polyclonal antibody a-syn-3 to human $\alpha$-Synuclein was previously described (Saha et al., 2000). The following antibodies were also used: rabbit polyclonal anti-TDP-43 antibody (10782-2-AP, Proteintech group, UK), rabbit polyclonal antiLC3B/MAP1LC3B antibody (NB600-1384, Novusbio), and rabbit polyclonal anti Beclin-1 antibody (H-300, Santa Cruz Biotechnology).

\section{Brain tissues}

For histological analysis, total of 37 samples were obtained post-mortem from individuals with AD (n=5), Pick disease (n=3), FTLD-MAPT P301L (n=2), FTLD-TDP (n=2), LBD (n=7), PSP (n=4) and CBD (n=5) as well as controls $(\mathrm{n}=9)$ as listed in Table 1. One FTLD-TDP case had a GRN (Granulin coding gene) mutation (deletion mutant of 812 to 815 ) or a C9orf72 (coding protein chromosome 9 open reading frame 72) mutation (stop codon in 268) (Table 1). Brain tissue samples obtained post-mortem from patients with these diseases were fixed in 10 $\%$ formalin solution and embedded in paraffin. Brain regions containing a high density of inclusions were histologically analysed in each case: $\mathrm{T} 1$ isocortex, frontal cortex and hippocampus in $\mathrm{AD}$, frontal cortex and hippocampus in Pick disease, frontal cortex and hippocampus in FTLD-P301L, hippocampus for FTLD-TDP, T1 isocortex, hippocampus, and substantia nigra in LBD, striatum in PSP and temporal isocortex in CBD. Control cases were non-demented individuals who died without known neurological disorders and the above regions were systematically analysed as negative control for pathological markers. Post-mortem tissues were also snap frozen in liquid nitrogen and kept at $-80{ }^{\circ} \mathrm{C}$ for biochemical analysis. The frozen tissues from frontal cortex from total of 31 cases were analysed by western blotting for biochemical analysis: control (n=8), AD ( $n=4)$, Pick disease (n=3), FTLD-MAPT P301L (n=2), FTLD-TDP (n=2), LBD (n=3), PSP (n=4) and CBD (n=5). The mean age at death and post-mortem delays of control and of pathological cases were not significantly different by unpaired t-test. Average age at death was $70.78+/-3.459$ and $70.86+/-1.516$ years (mean +/- SEM) for control $(n=9)$ and pathological cases $(n=28)$ respectively. Average post-mortem delays were $22.94+/-3.894$ hours and $32.59+/-3.242$ hours (mean +/- SEM) for control and pathological cases, respectively.

Most of the cases analysed in this study were enrolled in a brain donation program of the French national Brain Bank, GIE NeuroCEB (Bioresource Research Impact Factor number = BRIF BB-0033-00011) organized by a consortium of Patients Associations. An explicit consent had been signed by the patient or by the next of kin, in the name of the patient. The project was approved by the scientific committee of the Brain Bank. The consent form had been reviewed and accepted by the Ethical Committee "Comité de Protection des Personnes Paris Ile de France VI". The whole procedure has been certified by AFNOR (Association Française de Normalisation). The collection of post mortem samples has been declared to the Ministry of Research and Higher Education as requested by the French law and the Brain Bank has been officially authorized to provide sample to scientists (agreement AC-2013-1887). Some brain samples were collected and stored in the brain bank of the LHNN and 
the study using these post-mortem tissues performed in compliance and following approval of the Ethical Committee of the Medical School of the Free University of Brussels.

\section{Preparation of brain homogenates for biochemical analysis}

About $200 \mathrm{mg}$ of frontal isocortex was homogenised as reported (Ando et al., 2011; Ando et al., 2013) in 5 volumes of ice-cold modified RIPA buffer constituted of $50 \mathrm{mM}$ Tris pH 7.4 containing $150 \mathrm{mM} \mathrm{NaCl}, 1 \%$ NP40, $0.25 \%$ sodium deoxycholate, $5 \mathrm{mM}$ EDTA, $1 \mathrm{mM}$ EGTA, Roche complete protease inhibitors, $1 \mathrm{mM}$ PMSF, $25 \mu \mathrm{g} / \mathrm{ml}$ Pepstatin, $50 \mathrm{mM}$ Beta Glycerophosphate, $10 \mathrm{mM}$ sodium pyrophosphate, $10 \mathrm{mM}$ sodium fluoride, and phosphatase inhibitor cocktail 2, (SIGMA P-5726) and incubated for 60 min at $4{ }^{\circ} \mathrm{C}$ on a rotator. $100 \mu \mathrm{l}$ of homogenate was added with Laemmli sample buffer, sonicated on ice and kept at $-80{ }^{\circ} \mathrm{C}$ as total fraction. The rest of the homogenate was centrifuged $\left(16,100 \mathrm{x} g\right.$ for 20 minutes at $\left.4^{\circ} \mathrm{C}\right)$ and the supernatant was used as a RIPA soluble fraction. The RIPA-insoluble pellet was re-suspended in 5-fold volume of $8 \mathrm{M}$ urea supplemented with $5 \mathrm{mM}$ EDTA, $1 \mathrm{mM}$ EGTA, Roche complete protease inhibitors, $1 \mathrm{mM}$ PMSF, $25 \mu \mathrm{g} / \mathrm{ml}$ Pepstatin, $10 \mathrm{mM}$ sodium pyrophosphate, $10 \mathrm{mM}$ sodium fluoride, and phosphatase inhibitor cocktail 2 (P-5726, SIGMA) by vortex and sonication on ice and incubated for $30 \mathrm{~min}$ at room temperature on a rotator. The mixture was centrifuged at $16,100 \mathrm{xg}$ at $4^{\circ} \mathrm{C}$ for 20 minutes. The supernatant was used as RIPA insoluble fraction.

\section{Western blot}

Protein concentrations were estimated by the Bradford method (Bio-Rad). Samples $(20 \mu \mathrm{g} / \mathrm{lane})$ were run in 10 $\%$ or $15 \%$ of Tris-glycine gels depending on the molecular weight of the protein of interest and transferred onto PVDF membranes (Bio-Rad). The PVDF membranes were blocked in $10 \%$ (w/v) semi fat dry milk in TBS (Tris-buffered saline, $20 \mathrm{mM}$ Tris $\mathrm{pH} 7.6,150 \mathrm{mM} \mathrm{NaCl}$ ) for $1 \mathrm{~h}$ at room temperature and were incubated with primary antibodies overnight at $4^{\circ} \mathrm{C}$. After three times of rinses with TBS-T (TBS supplemented with $0.05 \%$ Tween 20), the membranes were incubated with anti-rabbit (G-21234, Invitrogen) or anti-mouse (G-21040, Invitrogen) immunoglobulin conjugated with horseradish peroxidase. After three rinses with TBS-T, the membranes were incubated with SuperSignal West Pico Substrate (Pierce) and were exposed to an X-ray film (Pierce) or to a DARQ-7 CCD cooled camera (Vilber-Lourmat) in a SOLO 4S WL system. Levels of proteins were estimated by densitometry analysis using the NIH Image J program, and adjusted for protein loading based on western blots performed with an anti-actin antibody.

\section{Histological staining and immunohistochemistry}

Formalin-fixed brain tissues were dehydrated, paraffin embedded and sliced into four- $\mu \mathrm{m}$ thick sections. For immunolabelling with the $\mathrm{ABC}$ method, deparaffinised and rehydrated tissue sections were heated in citrate buffer for $20 \mathrm{~min}$, incubated in $\mathrm{H}_{2} \mathrm{O}_{2}$ to inhibit endogenous peroxidase, rinsed in water and then incubated with a blocking solution containing $2 \%$ bovine serum albumin in TBS. Sections were then incubated overnight with the primary antibody. Adjacent tissue sections were sequentially incubated with the antibodies against PICALM, tau, $\alpha$-Synuclein or TDP-43 in order to compare these immunoreactivities in similar structures present in the adjacent tissue sections. The presence of the primary antibody was visualized with the Dako REAL detection system (which includes both anti-rabbit and anti-mouse secondary antibodies) using DAB as chromogen, followed by a nuclear counterstaining with haematoxylin. The slides were examined with a bright field 
microscope (Olympus Bx41) connected to an AxioCam I CC1 camera (Zeiss). To check for the specificity of PICALM labelling, anti-PICALM HPA019053 antibody was pre-incubated with $15 \mu \mathrm{g} / \mathrm{ml}$ of GST-PICALM (157H00008301, Tebu-bio) on a rotator at $4{ }^{\circ} \mathrm{C}$ overnight prior to immunohistochemistry as previously described (Ando et al., 2013). Double immunofluorescent labelling was carried out using goat anti-rabbit antibody conjugated with biotin (BA-1000, Dako) and goat anti-mouse antibody conjugated to Alexa 568 (A11031, Invitrogen). For PICALM staining, TSA TM Fluorescein System (NEL 701A00KT, Perkin Elmer) was used for amplification. The slides were mounted with Fluoromount-G (Southern biotech) and were observed with an upright confocal microscope (Olympus Fluoview Fv1000) or with an Axiovert 200M microscope (Zeiss) equipped with an ApoTome system (Zeiss).

\section{Co-localisation estimation}

The proportion of NFTs showing a co-localisation of tau and PICALM immunoreactivities was estimated on double immunostained slides in the affected brain areas of AD, Pick disease, PSP and LBD with Braak VI tau pathology. The percentage of PICALM positive NFTs was estimated by dividing the number of tau and PICALM positive NFTs by the number of total NFTs (tau positive). Five distinct zones on each slide were analysed for each disease case using a $20 \mathrm{x}$ magnification objective and an average percentage was calculated.

\section{Statistical analysis}

Statistical significance of comparisons between control subjects and patients with different diseases were determined by unpaired t-test for age and post-mortem delays, one-way ANOVA (with Dunnett's multiple comparison tests) and the correlations analyses were computed by Pearson correlation test (Prism 4 software, Graphpad). Values of $\mathrm{P}<0.05$ were considered significant. Histograms represent means +/- SEM. 


\section{Results}

We first started by performing immunolabellings against PICALM in post-mortem human tissue samples from control subjects and from neurodegenerative diseases. A strong PICALM immunoreactivity was detected in endothelial cells in blood vessels in all the cases (Fig S1B). The specificity of the immunolabelling for PICALM was confirmed by pre-incubating with recombinant PICALM protein that strongly decreased/abolished the labelling of endothelial, neuronal and microglial cells (Fig. S1C) (Ando et al., 2013). Omitting the primary HPA019053 PICALM antibody in the immunolabelling procedure resulted in no labelling in all cases (Fig S1D).

\section{PICALM immunoreactivity in a certain population of NFTs of AD and PSP and Pick bodies}

As previously reported in AD and in control subjects (Ando et al., 2013), neurons without NFTs contained weak diffuse cytoplasmic and perinuclear PICALM staining (Fig S1A). In AD, the same labelling was observed in neurons without NFTs. By contrast, NFT-bearing neurons, identified on adjacent sections, showed both strong tau and PICALM immunoreactivities (Fig. 1A, B). More than $85 \%$ of NFTs had a strong immunoreactivity for PICALM (Ando et al., 2013). PICALM immunoreactivity was detected in tau-positive Pick bodies of Pick disease cases (Fig. 1C, D). Some tau-positive NFTs in PSP were PICALM positive (Fig. 1E, F), while taupositive tufted astrocytes were PICALM negative (Fig. 1G, H). Coiled bodies were PICALM positive. In FTLDMAPT P301L, PICALM was not detected in NFTs (Fig. 1I, J). The characteristic tau-positive astrocytic plaques as well as the NFTs of CBD were PICALM negative (Fig 1K, L). PICALM immunoreactivity was not detected in TDP-43 positive inclusions in FTLD-TDP cases (Fig. 1M, N). Some of the LBD cases analysed in this study had mixed pathology with both Lewy bodies and AD related pathology, allowing to compare them for their PICALM immunreactivities: $\alpha$-synuclein positive inclusions were PICALM negative (Fig. 1O, P) but NFTs of LBD cases with tau pathology showed PICALM immunoreactivity (Fig. 2P-R). Microglial cells were PICALM positive in all cases. PICALM immunoreactivity in microglial cells was marked in Pick disease and FTLDMAPT P301L (not shown).

\section{Co-localisation of PICALM and phosphorylated tau in AD, Pick disease and PSP}

Double immunostaining for PICALM and anti-phosphotau antibodies (AT8 and PHF1) showed a co-localisation of PICALM and phosphotau in Pick bodies of Pick disease (Fig. 2A-C). About $65 \%$ of granule cells in the dentate gyrus of Pick disease brain had diffuse immunoreactivity for PICALM. PICALM staining was diffuse and weak but a corona of strong PICALM immunoreactivity was present at the periphery of Pick bodies (Fig. 2A-C). In PSP cases, both coiled bodies (Fig. 2 D-F) and NFTs (Fig. 2G-I) in the striatum showed a complete co-localisation of PICALM and phosphotau immunoreactivies. The frequency of PICALM positive NFTs of PSP were however estimated less than $20 \%$. PICALM immunoreactivity was generally less intense in NFTs of PSP than in NFTs found in AD brains. No immunoreactivity of PICALM was observed in the tufted astrocytes of PSP cases (Fig. 2J-L). In CBD cases, both astrocytic plaques (Fig. $2 \mathrm{M}-\mathrm{O}$ ) and NFTs (data not shown) were PICALM negative. In LBD cases with Braak VI tau pathology, NFT (but not Lewy bodies, see above) were PICALM positive (Fig. 2 P-R). About $60 \%$ of NFTs in the cases of LBD with some degree of tau pathology had co-localisation with PICALM in the entorhinal cortex.

\section{PICALM is associated with both $3 R$ and $4 R$ tau in tau-positive inclusions}


Since the co-localisation of PICALM and tau was not observed in tufted astrocytes of PSP, astrocytic plaques of CBD or in NFTs of CBD cases, we next analysed whether there might be a tau isoform specific interaction with PICALM. A double immunofluorescent staining for PICALM and RD3 or RD4 antibodies specific for 3R or 4R tau respectively showed that PICALM was associated with both 3R (Fig. 3 A-C) and 4R tau (Fig. 3 D-F) in NFTs of AD brains. In Pick bodies, 3R tau co-localised with PICALM (Fig. 3 G-I). 4R tau and PICALM were co-localised in the NFTs of PSP cases (Fig. 3 J-L).

Levels of RIPA-soluble PICALM are decreased in tauopathies and are inversely correlated with levels of phosphorylated tau

In order to compare the levels of PICALM protein and solubility, the frontal cortex was homogenized in RIPA buffer, fractionated and analysed by western blotting in control, AD, Pick disease, FTLD-MAPT P301L mutation, FTLD-TDP, LBD (all the three LBD cases analysed for western blotting in this study had Braak VI tau pathology), PSP and CBD subjects (Fig. 4A). In the total fraction, mean levels of phosphorylated tau were significantly increased in AD, Pick disease, FTLD-MAPT P301L, LBD and CBD cases (Fig. 4B). The level of PICALM was significantly decreased in AD, Pick disease, FTLD-MAPT P301L and CBD cases (Fig. 4C). The levels of PICALM were inversely correlated with the levels of phosphorylated tau with a high degree of significance in the total fraction (Fig 4D).

The homogenates were further fractionated by centrifugation. In the RIPA soluble fraction, the level of PICALM was altered (Fig. 5A). PICALM, especially the longest isoform, was significantly decreased in the soluble fraction of AD, Pick disease, FTLD-MAPT P301L, FTLD-TDP, LBD, PSP and CBD cases (Fig. 5C). In the RIPA insoluble fraction (Fig 5B), PICALM levels were significantly increased in AD cases but not in other diseases (Fig 5D). RIPA soluble and RIPA-insoluble phosphorylated tau was absent in controls and in FTLDTDP, but was significantly increased in AD, Pick disease, FTLD-MAPT P301L mutation, LBD and CBD and was less abundant in PSP cases (not shown). The ratio of RIPA-soluble/insoluble PICALM (Fig.5E) was significantly decreased in all the analysed cases except in PSP that showed a non-significant decrease. The ratios of soluble/insoluble PICALM levels were negatively correlated with the levels of phosphorylated tau (Fig 5F).

\section{PICALM levels are negatively correlated with the levels of the autophagy related proteins LC3-II and}

\section{Beclin-1}

PICALM downregulation has recently been reported to inhibit both autophagosome synthesis and degradation in cellular and animal models (Moreau et al., 2014). In order to investigate the association of solubility change and protein level of PICALM with autophagy, levels of autophagy markers LC3-II and Beclin-1 were analysed in human neurodegenerative diseases. When LC3-I is converted to LC3-II, LC3-II is conjugated with phosphatidylethanolamine and is attached to the surface of autophagosomes. Augmented LC3-II level can be related to either increased autophagosome synthesis or reduced autophagosome turnover. Level of LC3-II was measured by normalizing with actin signal in accordance with guidelines for autophagy monitoring (Klionsky et al., 2016). The level of LC3-II was generally increased in the disease cases but a highly significant increase of the LC3-II/actin ratio was observed in AD and LBD with Braak VI tau pathology cases (Fig. 6A, B). Beclin-1 is a key component of autophagy involved in recruitment of membranes to autophagosomes. A significant decrease of Beclin-1 was also observed in the neurodegenerative diseases except PSP (Fig 6C). A highly significant 
correlation was found between decreased levels of PICALM and increased levels of LC3-II ( $\mathrm{p}=0.0032)$ or decreased levels of Beclin-1 ( $\mathrm{p}=0.0295)$ in the total brain lysates from these diseases (Fig 6D,E). Furthermore, a statistically more significant correlation was found between soluble PICALM protein level and LC3-II $(\mathrm{p}=0.0023)$ or Beclin-1 ( $\mathrm{p}=0.0002)($ Fig $6 \mathrm{~F}, \mathrm{G})$. Taken together, these data suggest that PICALM reduction, especially in the soluble pool, may be associated with the abnormalities in autophagy function in these neurodegenerative diseases. 


\section{Discussion}

In the present study, the levels of PICALM and its association with tau pathology and its correlation with autophagy markers were analysed in several tauopathies and other neurodegenerative diseases. Immunohistochemistry showed an association of PICALM with tau inclusions in AD (NFTs), in PSP (NFTs and coiled bodies), and in Pick disease (Pick bodies). NFTs and astrocytic plaques of CBD were PICALM immunonegative as well as the astrocytic tufts observed in PSP and the inclusions seen in FTLD-MAPT P301L mutation. PICALM immunoreactivity was not linked to the isoforms of tau (3R or 4R). Lewy bodies and TDP inclusions were also negative. Endothelial cells were PICALM immunopositive - a positivity which can be used as an internal control. Western blots revealed a decrease in full-length PICALM measured in the RIPA soluble fraction in all the cases. The decrease of soluble PICALM protein was negatively correlated with increased phosphotau levels in these diseases. In AD and LBD with Braak VI tau pathology, the autophagosome marker LC3-II was increased. Beclin-1 was decreased in all disorders but PSP. Significant correlations were observed between the levels of soluble PICALM and these autophagy markers.

\section{Disease and cell-type specific association between PICALM and tau}

PICALM was accumulated in the NFTs of $\mathrm{AD}$, the Pick bodies of Pick disease, coiled bodies, a certain population of the NFTs of PSP and NFTs of LBD with Braak VI tau pathology. PICALM was co-localised with phosphorylated tau in these inclusions. The association of PICALM with protein aggregates in cellular inclusions was specific for tau-positive inclusions since we did not observe any PICALM immunoreactivity in tau-negative inclusions such as Lewy bodies or TDP-43 positive inclusions. PICALM was associated with both $3 \mathrm{R}$ and $4 \mathrm{R}$ tau isoforms in tau-positive inclusions, since PICALM co-localised with both 3R and 4R tau in NFTs in $\mathrm{AD}$, and either 3R tau or 4R tau in Pick's disease and PSP, respectively. PICALM was present in neuronal and oligodendroglial tau-positive inclusions but was not detected in tau positive tufted astrocytes in PSP or astrocytic plaques in CBD. This observation is consistent with our previous report showing that PICALM immunoreactivity was not significantly detected in the astrocytes in control and AD brains (Ando et al., 2013), suggesting that absence of recruitment of PICALM in tau-positive inclusions in astrocytes reflects its low level of expression in these cells. An alternative explanation is that differential recruitment of PICALM in tau-positive inclusion reflects distinct processing and post-translational modifications of tau in diverse cell types and in various tauopathies. Indeed, we could not detect any PICALM immunoreactivity in tau pathology in FTLDMAPT P301L or CBD, whereas PICALM was detected in a certain population of NFTs and coiled bodies in PSP. A different proteolytic processing of abnormal tau in PSP and CBD has been reported (Arai et al., 2004), and this might lead to difference in PICALM/tau interaction in these diseases. The degree of tau aggregation might also underlie the extent of PICALM/tau interaction. For instance, pretangles are the characteristics of FTLD-MAPT P301L and may thus explain the absence of co-localisation of phosphorylated tau and PICALM (Spillantini et al., 1998). Tau extracted from CBD or PSP has distinct electrophoretic migration patterns from AD PHF (Sergeant et al., 1999). Indeed filamentous structures, seen at the ultrastructural level, are more densely packed in AD than in FTLD-MAPT P301L (Spillantini et al., 1998) or in CBD (Tracz et al., 1997). Filamentous tau aggregation is more often observed in NFTs and coiled bodies in PSP, whereas there is less fibrillar tau aggregation in CBD (Uchihara, 2014; Yoshida, 2014). We also previously observed that PICALM immunoreactivity was not frequently associated to pretangles in $\mathrm{AD}$ (Ando et al., 2013), - an observation that 
corroborates the hypothesis of PICALM being preferentially associated with fibrillar tau. Some differences in the PICALM/tau association between tauopathies might also be related to disease specific tau misfolding and templating (Clavaguera et al., 2013; Sanders et al., 2014). Disease-specific PICALM/tau association in AD, trisomy 21, Pick disease, PSP and LBD with Braak VI tau pathology could thus be related to a tau-conformer specific interaction with protein partners such as PICALM.

The molecular basis for the PICALM-tau interaction in inclusions might be direct or indirect. The estimated isoelectric point (pI) of PICALM whole protein (isoform 1 with 652 amino acids) is 7.70 (http://web.expasy.org/cgi-bin/compute_pi/pi_tool): N-terminal ANTH domain (amino acids 20 to 289) is cationic ( $\mathrm{pI}=9.07$ ) but $\mathrm{C}$ terminus contains highly anionic residues around amino acids 521-560 (pI=4.54). The anionic nature of PICALM C terminus might lead to an electrostatic interaction with the positively charged repeat domain of tau, whereas the cationic N-terminal ANTH domain of PICALM might interact with the negatively charged N-terminal 125 amino acids tau domain. The anionic PICALM C terminus might also favour tau fibrillization, since polyanions have been reported to be powerful inducers of tau fibrillization (Goedert et al., 1996). Interestingly, it has been reported that $\mathrm{PIP}_{2}(4,5)$ is enriched in NFTs and GVDs (Nishikawa et al., 2014). PICALM directly interacts with $\mathrm{PIP}_{2}$ via its N-terminal ANTH domain (Ford et al., 2001), and PICALM association to NFTs might be driven by this interaction. Also, tau N-terminal projection domain interacts with membrane structures (Brandt et al., 1995). The complex of tau, membrane and membrane-associated proteins such as PICALM might be misregulated in pathological conditions. This hypothesis may be supported by the studies from other teams on general abnormalities in endocytic machinery proteins in AD (Coleman and Yao, 2003; Yao, 2004).

PICALM was associated to microglial cells, as previously reported, and increased PICALM positive microglial cells generally reflected increased microgliosis associated to these diseases, which might be related to a previous report of increased level of PICALM transcripts in AD brain (Baig et al., 2010).

\section{Decreased soluble PICALM level in tauopathies.}

We have shown previously that PICALM is cleaved and decreased in AD brains (Ando et al., 2013). In this study, we report that levels of PICALM were highly reduced in the RIPA soluble fractions of neurodegenerative disorders other than AD. Since PICALM is a substrate of calpain and caspase (Kim and Kim, 2001; Rudinskiy et al., 2009; Ando et al., 2013), this reduction of RIPA soluble PICALM may be related to calpain and/or caspase activation in these neurodegenerative disorders (Saito et al., 1993; Adamec et al., 2002), leading to rapid proteolysis of PICALM. A pool of RIPA insoluble PICALM was detected in control subjects and in neurodegenerative disorders, but its level, although tending to increase in tauopathies, was not significantly different from controls, suggesting that a proportion of RIPA insoluble PICALM does not result from a pathological process. A fraction of RIPA insoluble PICALM may indeed derive from endothelial tissues in blood vessels where PICALM is highly expressed (Baig et al., 2010; Zhao et al., 2015). The ratio of RIPA soluble/insoluble PICALM was nevertheless quite significantly decreased in tauopathies, indicating a shift towards increased insolubility of PICALM in these disorders. This shift could result from the recruitment of PICALM into insoluble tau-positive inclusions in which PICALM and phosphorylated tau are co-localised. The soluble/insoluble PICALM ratio was decreased not only in tauopathies but also in cases with FTLD-TDP, suggesting that it was a general phenomenon rather than only the recruitment of PICALM into insoluble tau- 
positive inclusions. A reduction of PICALM levels might have severe consequences on endocytic functions, leading to dysregulation of membrane dynamics of endo-lysosomal components (Nixon, 2005). A decrease of PICALM in the microvessels fraction in AD brain was recently reported, with evidence that reduction of PICALM in endothelial cells decreases Aß clearance and transcytosis through the blood-brain barrier (Zhao et al., 2015). Interestingly, acute reduction of PICALM expression by siRNA was also shown to induce a significant dendritic retraction in hippocampal neuronal primary culture (Bushlin et al., 2008).

We found that there was a significant negative correlation between the levels of PICALM and PHF1 positive phosphorylated tau in the total fraction and in the RIPA soluble fraction of tauopathies. This suggests that the abnormal processes leading to reduction of PICALM and to phosphorylation of tau in these diseases are occurring in parallel or are causally linked. Since decrease of PICALM impairs autophagy and leads to accumulation of phosphorylated tau in a zebrafish model expressing enhanced GFP (EGFP)-tagged human tau under the control of the rhodopsin promoter (Moreau et al., 2014), this negative correlation might reflect autophagy impairment developing in tauopathies as a result of PICALM decrease.

The nature of upstream factors affecting PICALM expression are still poorly understood but might be related to specific functional PICALM variants. A $\mathrm{B}$ does not appear to be an upstream factor since it does not accumulate in the tauopathies in which PICALM levels were decreased - with the sole exception of AD.

\section{Decreased PICALM level and impairment of autophagy}

PICALM modulates autophagy by regulating endocytosis of SNAREs, acting at the levels of both autophagosome biogenesis and autophagosome degradation (Moreau et al., 2014). Autophagy is a key player in neurodegenerative disorders where protein aggregates accumulate (Nixon, 2013). PICALM was reported to be a modulator of autophagy, affecting both A $\beta$ production (Tian et al., 2013; Tian et al., 2014) and tau clearance (Moreau et al., 2014). Knocking down PICALM expression in cultured cells induces accumulation of autophagy substrates and intracellular protein aggregates of phosphorylated tau, mutant huntingtin and p62 (Moreau et al., 2014). We found a significant inverse correlation between decreased levels of PICALM and increased levels of the autophagosomal marker LC3-II in brain tissues. Increased levels of LC3-II have been observed in experimental conditions with increased autophagosomes accumulation, e.g. due to impairment of their lysosomal clearance (Boland et al., 2008). Accumulation of autophagic vacuoles is prominent in AD and thought to reflect principally a defect of autolysosomal proteolysis (Nixon and Yang, 2011). Our result showing increased levels of LC3-II suggests that this accumulation of autophagic vacuoles also occurs in LBD with Braak VI tau pathology.

Beclin-1 plays a key role in autophagy and is involved in the recruitment of membranes to generate autophagosomes. Levels of Beclin-1 are decreased in the cortex in AD (Pickford et al., 2008). We confirmed the decrease of Beclin-1 in AD and found also a significant decrease in several other tauopathies (Pick disease, FTLD-MAPT P301L, FTLD-TDP, LBD with Braak VI tau pathology and CBD). This decrease of Beclin-1 was significantly correlated with decreased PICALM levels.

Altogether, our results suggest that decreased levels of PICALM should be associated with impairment of autophagy at different levels of the autophagy pathway in several neurodegenerative diseases.

\section{GWAS revealed genetic LOAD risk factors and tau pathology}


Genetic association studies have revealed numbers of LOAD-susceptible genetic loci (Lambert et al., 2013). How each of these genes from these loci is involved in LOAD aetiology remains challenging to elucidate. For instance, APOE allele 4 is the most significant genetic risk factor for LOAD and seems to affect clearance and oligomerization of $\mathrm{A} \beta$ as well as tau aggregation (Huang and Mucke, 2012). We noticed a striking similarity between APOE and PICALM in term of association with tau in AD and in other tauopathies. APOE4 is aberrantly cleaved and its cleaved fragments interact with tau in AD brains (Huang et al., 2001; Rohn et al., 2012). APOE immunoreactivity is also found in tau positive inclusions of other tau-related neurodegenerative disorders such as Pick disease (Farrer et al., 1995; Hayashi et al., 1998; Rohn et al., 2013). PICALM and APOE risk alleles are associated with brain atrophy and cognitive function in LOAD patients (Morgen et al., 2014). A recent study has shown that another LOAD genetic risk factor, BIN1, directly binds to tau (Chapuis et al., 2013). Variants of the clusterin gene $(C L U)$ have also been identified as a LOAD genetic risk factor and iCLU, an intracellular form of clusterin, interacts with tau (Zhou et al., 2014). Tau spreading in tauopathies implying transmission from cell to cell relies on internalisation of tau by different mechanisms that remain to be deciphered, but since both BIN1 and PICALM are key molecules regulating endocytosis, they might also be involved in modulating tau spreading. Altogether, these studies and our results suggest that several risk factors found in LOAD by GWAS might affect disease progression by interacting with tau processing.

In conclusion, our immunohistochemical and biochemical data indicate that PICALM is co-localised in tau positive inclusions in AD, Pick disease, PSP and NFTs of LBD with Braak VI tau pathology and may interacts with both $3 \mathrm{R}$ and $4 \mathrm{R}$ tau, that soluble PICALM level is generally decreased in tauopathies and is correlated with changes in autophagy markers. PICALM abnormal processing thus seems involved not only in AD but also in other tauopathies and neurodegenerative disorders. 
Acknowledgements: This study was supported by INSERM: C.D was supported by ANR Franco-Canada CholAD (U975-P-A1L4-RP-RPV10014DDA-R10178DD), by MassImage (U975-P-A1L4-RP-RPV10015DDAR10179DD), and by "Investissements d'avenir" (ANR-10-IAIHU-06). J.P.B was supported by grants from the Belgian Fonds de la Recherche Scientifique Médicale (T.0023.15), the Fund Aline (King Baudoin Foundation), the Belgian Fondation Recherche Alzheimer/ Stichting Alzheimer Onderzoek (14001), the Génicot Fund (ULB) and this work was performed in the frame of the IAP program (P7/16) of the Belgian Federal Science Policy Office. K.A was a fellowship recipient of the French "Fondation Plan Alzheimer" and is a research fellow of the Belgian F.N.R.S. The brain tissues were provided by the GIE NeuroCEB brain bank (funded by France Alzheimer, France Parkinson, ARSEP, CSC patients Associations) and the LHNN brain bank. We thank Dr Peter Davies for providing PHF1 antibody and Dr. Sabrina Turbant for human tissue preparation. The authors acknowledge Dr. Fabrice Gankamk for valuable advice on autophagy marker detection. Analysis by confocal microscopy was performed at the cellular imaging platform of Pitié-Salpêtrière PICPS. 


\section{Legends of table and figures}

\section{Table 1: Human cases analysed in this study.}

The neuropathological staging of neurofibrillary pathology was determined according to Braak staging (Braak and Braak, 1991) and staging for amyloid plaque scores according to Thal (Thal et al., 2002). AD: Alzhemer disease. P301L: FTLD-MAPT (microtubule associated protein tau) mutation at P301L. TDP: FTLD with TDP-43 positive inclusions. GRN: Granulin coding gene. C9: C9orf72 coding gene. LBD: Lewy body disease, diffuse type. PSP: progressive supranuclear palsy. CBD: corticobasal degeneration.

PMD: post-mortem delay. NA: not available. Paraffin embedded tissues of affected brain regions were analysed by immunohistochemistry (IHC). Frozen tissues of frontal isocortex were analysed by western blotting (WB).

\section{Figure 1: PICALM immunoreactivity in neuronal and glial inclusions in tauopathies.}

A and B: Immunolabelling on adjacent sections for phosphorylated tau (A) (PHF1 antibody) and for PICALM (B) in temporal isocortex from AD. PICALM and phosphotau immunoreactivity were detected in the same NFT. $\mathrm{C}$ and D: Immunolabelling on adjacent sections for phosphorylated tau (C) and for PICALM (D) in hippocampal dentate gyrus from Pick disease (PiD). Pick bodies in granule cells are both PICALM and phosphotau positive.

E-H: Immunolabelling on adjacent sections for phosphorylated tau (E) and for PICALM (F) in striatum from PSP. A globular NFT shows both phosphotau (E) and PICALM (F) immunoreactivity on adjacent sections. Taupositive tufted astrocytes are phosphotau positive (G) but PICALM negative (H).

I-J: Immunolabelling on adjacent sections for phosphotau (I) and for PICALM (J) in frontal cortex from FTLDMAPT P301L case. NFTs are phosphotau positive but PICALM negative.

K-L: Immunolabelling on adjacent sections for phosphotau (K) and for PICALM (L) in temporal isocortex from CBD. Astrocytic plaques are phosphotau positive but PICALM negative.

M-N: Immunolabelling on adjacent sections for TDP-43 (M) and for PICALM (N) in of temporal isocortex of FTLD-TDP case. TDP-43 positive inclusions are PICALM negative.

O-P: Immunolabelling on adjacent sections for $\alpha$-Synuclein $(\mathrm{O})$ and for PICALM (P) in substantia nigra from a LBD case. Lewy bodies are $\alpha$-Synuclein positive but PICALM negative.

Scale bars: $10 \mu \mathrm{m}$.

Figure 2: PICALM and tau are co-localised in the inclusions in Pick disease, PSP and LBD with tau pathology but not in CBD.

A-C: Double immunofluorescence for phosphotau (A) (PHF1 antibody) and PICALM (B) in Pick disease.

PICALM immunoreactivity co-localises with phosphotau in Pick bodies in CA1 pyramidal cells in the hippocampus (merge in $\mathrm{C}$ ).

D-L: Double immunofluorescence for phosphotau (D,G,J) (AT8 antibody) and PICALM (E,H,K) in PSP. PICALM immunoreactivity co-localises with phosphotau in coiled bodies (D and E, merge in F), and in NFTs ( $\mathrm{G}$ and $\mathrm{H}$, merge in $\mathrm{I}$ ) in striatum. PICALM immunoreactivity does not co-localise with phosphotau in tufted astrocytes ( $\mathrm{J}$ and $\mathrm{K}$, merge in $\mathrm{L}$ ).

M-O: Double immunofluorescence for phosphotau (M) (AT8 antibody) and PICALM (N) in CBD. PICALM immunoreactivity does not co-localise with phosphotau in astrocytic plaques (merge in $\mathrm{O}$ ). 
P-R: Double immunofluorescence for phosphotau (P) (PHF1 antibody) and PICALM (Q) in mixed LBD with Braak VI tau pathology. PICALM immunoreactivity co-localises with phosphotau in NFTs in LBD (merge in $\mathrm{R})$.

Scale bar: $10 \mu \mathrm{m}$.

Figure 3: Both 3R and 4R tau isoforms co-localise with PICALM in tau-positive inclusions

A-F: Double immunofluorescence for 3R tau (A) (RD3 antibody) and PICALM (B) (merge in C) or for 4R tau (D) (RD4 antibody) and PICALM (E) (merge in F) in AD. Both 3R and 4R tau were co-localised with PICALM in NFTs in AD temporal isocortex.

G-I: Double immunofluorescence for 3R tau (G) and PICALM (H) in Pick disease. 3R tau is co-localised with PICALM in Pick bodies (merge in I).

J-L: Double immunofluorescence for 4R tau (J) and PICALM (K) in PSP. 4R tau is co-localised with PICALM in NFTs of PSP (merge in L).

Scale bar: $10 \mu \mathrm{m}$.

Figure 4: Levels of PICALM are decreased and of phosphotau are increased in the total fraction of frontal cortex in AD, Pick disease, FTLD-MAPT P301L and CBD

A: Representative western blots for PICALM, PHF1 and actin in total fractions from homogenates of frontal cortex from control, Pick Disease, FTLD-MAPT P301L, FTLD-TDP, LBD with Braak VI tau pathology, PSP and CBD cases. Arrowhead shows a $50 \mathrm{kDa}$ cleaved PICALM fragment.

B and C: Quantification of the levels of PHF1 positive tau (B) and of PICALM (C) normalised to actin in the total fraction of frontal cortex from control $(n=8), A D(n=4)$, Pick Disease ( $n=3)$, FTLD-MAPT P301L $(n=2)$, FTLD-TDP ( $n=2), \operatorname{LBD}(n=3), \operatorname{PSP}(n=4)$ and CBD $(n=5)$ cases. A significant reduction of PICALM is observed in AD, Pick disease, FTLD-MAPT P301L and CBD. $\left({ }^{*} p<0.05\right.$, ***p $<0.001$ by one-way ANOVA and Dunnett's test).

D: Correlation analysis between the levels of PICALM and of PHF1 positive phosphotau both normalised to actin in the total fraction. There is a significant negative correlation between PICALM and phosphotau levels $(\mathrm{r}=-0.57, \mathrm{n}=31, \mathrm{p}=0.0008$, by Pearson correlation test $)$.

Figure 5: Soluble PICALM is decreased in neurodegenerative diseases and the solubility change is correlated with levels of phosphotau.

A-B: Representative western blots for PICALM and actin in RIPA soluble fraction (A) and RIPA insoluble fractions (B) (same cases) of frontal cortex from control, Pick Disease, FTLD-MAPT P301L, FTLD-TDP, LBD with Braak VI tau pathology, PSP and CBD cases. Arrowhead shows a $50 \mathrm{kDa}$ cleaved PICALM fragment.

C-D: Quantification of the level of soluble (C) and insoluble (D) PICALM normalised to actin in control $(\mathrm{n}=8)$, AD ( $\mathrm{n}=4$ ), Pick Disease ( $\mathrm{n}=3$ ), FTLD-MAPT P301L ( $\mathrm{n}=2$ ), FTLD-TDP ( $\mathrm{n}=2)$, LBD with Braak VI tau pathology $(n=3)$, PSP $(n=4)$ and CBD $(n=5)$ cases. A significant reduction of soluble PICALM is observed in all the neurodegenerative disease cases. A significant increase of RIPA insoluble PICALM is observed in AD $\left({ }^{*} p<\right.$ $0.05, * * p<0.01, * * * p<0.001$ by one-way ANOVA and Dunnett's test ). 
E: Quantification of the level of the ratio of soluble/insoluble PICALM of cases analysed in (C) and (D). The ratio of soluble/insoluble PICALM was significantly decreased in AD, Pick disease, FTLD-MAPT P301L, FTLD-TDP, LBD with Braak VI tau pathology and in CBD $(* p<0.05, * * p<0.01, * * * p<0.001$ by one-way ANOVA).

F: Correlation analysis between the ratios of soluble/insoluble PICALM and of PHF1 positive phosphotau normalised to actin. There is a significant negative correlation between the ratio of soluble/insoluble PICALM and phosphotau levels. $(* * \mathrm{p}=0.0013, \mathrm{r}=-0.5515, \mathrm{n}=31$, by Pearson correlation test).

Figure 6: Decreased levels of PICALM are correlated with increased levels of LC3-II and decreased levels of Beclin-1

A: Representative western blots for LC3, Beclin-1 and actin in total fraction from homogenates of frontal cortex from control, AD, Pick Disease, FTLD-MAPT P301L, FTLD-TDP, LBD with Braak VI tau pathology, PSP and CBD cases.

B. Quantification of the level of LC3-II normalised to actin in control (n=8), AD ( $n=4)$, Pick Disease ( $n=3)$, FTLD-MAPT P301L (n=2), FTLD-TDP (n=2), LBD (n=3), PSP $(n=4)$ and CBD $(n=5)$ cases. A significant increase of LC3-II is observed in AD and LBD cases (***p $<0.001$ by one-way ANOVA and Dunnett's test ).

C: Quantification of the level of Beclin-1 in the same subjects as in (B). A significant decrease of Beclin-1 is observed in AD, Pick disease, FTLD-MAPT P301L, FTLD-TDP, LBD with Braak VI tau pathology and CBD cases $(* p<0.05, * * p<0.01, * * * \mathrm{p}<0.001$ by one-way ANOVA and Dunnett's test $)$.

D-E: Correlation analysis between the levels of PICALM in the total fraction and LC3-II normalised to actin (D) and total PICALM and Beclin-1 normalised to actin (E), in the same subjects as in (B). A significant negative correlation is observed between levels of PICALM and LC3-II (** $\mathrm{p}=0.0032, \mathrm{r}=-0.5121, \mathrm{n}=31$, by Pearson correlation test) and a significant positive correlation between levels of PICALM and Beclin-1. $(* \mathrm{p}=0.0295$, $\mathrm{r}=0.39 \mathrm{n}=31$, by Pearson correlation test).

F-G: Correlation analysis between the levels of soluble PICALM and LC3-II normalised to actin (F) and soluble PICALM and Beclin-1 normalised to actin $(\mathrm{G})$, in the same subjects as in (B). A significant negative correlation is observed between levels of soluble PICALM and LC3-II (** $\mathrm{p}=0.0023, \mathrm{r}=-0.5277, \mathrm{n}=31$, by Pearson correlation test) and a significant positive correlation between levels of soluble PICALM and Beclin-1. (*** $\mathrm{p}=0.0002, \mathrm{r}=0.6140, \mathrm{n}=31$, by Pearson correlation test $)$.

\section{Supplementary Figure 1}

\section{Positive and negative controls for the PICALM immunolabelling}

A: PICALM immunolabelling in neurons in the hippocampus in a control subject. A weak and diffuse cytoplasmic and perinuclear staining is observed.

B: A strong PICALM immunoreactivity is detected in endothelial cells in blood vessels in a control brain tissue.

C: Pre-incubation of the PICALM antibody with recombinant PICALM protein strongly decreased the labelling of endothelial cells and of microglial cells.

D: Omission of the primary HPA019053 PICALM antibody in the immunolabelling procedure resulted in no labelling.

Scale bar: $20 \mu \mathrm{m}$. 


\section{References}

Adamec E, Mohan P, Vonsattel JP, Nixon RA. Calpain activation in neurodegenerative diseases: confocal immunofluorescence study with antibodies specifically recognizing the active form of calpain 2 . Acta neuropathologica 2002; 104(1): 92-104.

Ando K, Brion JP, Stygelbout V, Suain V, Authelet M, Dedecker R, et al. Clathrin adaptor CALM/PICALM is associated with neurofibrillary tangles and is cleaved in Alzheimer's brains. Acta neuropathologica 2013; 125(6): 861-78.

Ando K, Leroy K, Heraud C, Yilmaz Z, Authelet M, Suain V, et al. Accelerated human mutant tau aggregation by knocking out murine tau in a transgenic mouse model. The American journal of pathology 2011; 178(2): 80316.

Arai T, Ikeda K, Akiyama H, Nonaka T, Hasegawa M, Ishiguro K, et al. Identification of amino-terminally cleaved tau fragments that distinguish progressive supranuclear palsy from corticobasal degeneration. Annals of neurology 2004; 55(1): 72-9.

Baig S, Joseph SA, Tayler H, Abraham R, Owen MJ, Williams J, et al. Distribution and expression of picalm in Alzheimer disease. Journal of neuropathology and experimental neurology 2010; 69(10): 1071-7.

Boland B, Kumar A, Lee S, Platt FM, Wegiel J, Yu WH, et al. Autophagy induction and autophagosome clearance in neurons: relationship to autophagic pathology in Alzheimer's disease. J Neurosci 2008; 28(27): 6926-37.

Braak H, Braak E. Neuropathological stageing of Alzheimer-related changes. Acta neuropathologica 1991; 82(4): 239-59.

Brandt R, Leger J, Lee G. Interaction of tau with the neural plasma membrane mediated by tau's amino-terminal projection domain. The Journal of cell biology 1995; 131(5): 1327-40.

Buee L, Bussiere T, Buee-Scherrer V, Delacourte A, Hof PR. Tau protein isoforms, phosphorylation and role in neurodegenerative disorders. Brain research Brain research reviews 2000; 33(1): 95-130.

Bushlin I, Petralia RS, Wu F, Harel A, Mughal MR, Mattson MP, et al. Clathrin assembly protein AP180 and CALM differentially control axogenesis and dendrite outgrowth in embryonic hippocampal neurons. J Neurosci 2008; 28(41): 10257-71.

Chapuis J, Hansmannel F, Gistelinck M, Mounier A, Van Cauwenberghe C, Kolen KV, et al. Increased expression of BIN1 mediates Alzheimer genetic risk by modulating tau pathology. Molecular psychiatry 2013; 18(11): 1225-34.

Clavaguera F, Akatsu H, Fraser G, Crowther RA, Frank S, Hench J, et al. Brain homogenates from human tauopathies induce tau inclusions in mouse brain. Proceedings of the National Academy of Sciences of the United States of America 2013; 110(23): 9535-40.

Coleman PD, Yao PJ. Synaptic slaughter in Alzheimer's disease. Neurobiology of aging 2003; 24(8): $1023-7$. Dickson DW, Rademakers R, Hutton ML. Progressive supranuclear palsy: pathology and genetics. Brain Pathol 2007; 17(1): 74-82.

Duyckaerts C, Delatour B, Potier MC. Classification and basic pathology of Alzheimer disease. Acta neuropathologica 2009; 118(1): 5-36.

Farrer LA, Abraham CR, Volicer L, Foley EJ, Kowall NW, McKee AC, et al. Allele epsilon 4 of apolipoprotein E shows a dose effect on age at onset of Pick disease. Experimental neurology 1995; 136(2): 162-70.

Feany MB, Dickson DW. Neurodegenerative disorders with extensive tau pathology: a comparative study and review. Annals of neurology 1996; 40(2): 139-48.

Ford MG, Pearse BM, Higgins MK, Vallis Y, Owen DJ, Gibson A, et al. Simultaneous binding of PtdIns(4,5)P2 and clathrin by AP180 in the nucleation of clathrin lattices on membranes. Science (New York, NY 2001; 291(5506): 1051-5.

Goedert M, Jakes R, Spillantini MG, Hasegawa M, Smith MJ, Crowther RA. Assembly of microtubuleassociated protein tau into Alzheimer-like filaments induced by sulphated glycosaminoglycans. Nature 1996; 383(6600): 550-3.

Harold D, Abraham R, Hollingworth P, Sims R, Gerrish A, Hamshere ML, et al. Genome-wide association study identifies variants at CLU and PICALM associated with Alzheimer's disease. Nature genetics 2009; 41(10): 1088-93.

Hayashi S, Wakabayashi K, Iwanaga K, Kakita A, Seki K, Tanaka M, et al. Pick's disease: selective occurrence of apolipoprotein E-immunoreactive Pick bodies in the limbic system. Acta neuropathologica 1998; 95(1): 1-4. Huang Y, Liu XQ, Wyss-Coray T, Brecht WJ, Sanan DA, Mahley RW. Apolipoprotein E fragments present in Alzheimer's disease brains induce neurofibrillary tangle-like intracellular inclusions in neurons. Proceedings of the National Academy of Sciences of the United States of America 2001; 98(15): 8838-43.

Huang Y, Mucke L. Alzheimer mechanisms and therapeutic strategies. Cell 2012; 148(6): 1204-22.

Kim JA, Kim HL. Cleavage of purified neuronal clathrin assembly protein (CALM) by caspase 3 and calpain. Experimental \& molecular medicine 2001; 33(4): 245-50. 
Klionsky DJ, Abdelmohsen K, Abe A, Abedin MJ, Abeliovich H, Acevedo Arozena A, et al. Guidelines for the use and interpretation of assays for monitoring autophagy (3rd edition). Autophagy 2016; 12(1): 1-222.

Kovacs GG. Invited review: Neuropathology of tauopathies: principles and practice. Neuropathology and applied neurobiology 2015; 41(1): 3-23.

Lambert JC, Heath S, Even G, Campion D, Sleegers K, Hiltunen M, et al. Genome-wide association study identifies variants at CLU and CR1 associated with Alzheimer's disease. Nature genetics 2009; 41(10): 1094-9. Lambert JC, Ibrahim-Verbaas CA, Harold D, Naj AC, Sims R, Bellenguez C, et al. Meta-analysis of 74,046 individuals identifies 11 new susceptibility loci for Alzheimer's disease. Nature genetics 2013; 45(12): 1452-8. Lee VM, Goedert M, Trojanowski JQ. Neurodegenerative tauopathies. Annual review of neuroscience 2001; 24: 1121-59.

Mackenzie IR, Neumann M, Bigio EH, Cairns NJ, Alafuzoff I, Kril J, et al. Nomenclature for neuropathologic subtypes of frontotemporal lobar degeneration: consensus recommendations. Acta neuropathologica 2009; 117(1): 15-8.

Moreau K, Fleming A, Imarisio S, Lopez Ramirez A, Mercer JL, Jimenez-Sanchez M, et al. PICALM modulates autophagy activity and tau accumulation. Nature communications 2014; 5: 4998.

Morgen K, Ramirez A, Frolich L, Tost H, Plichta MM, Kolsch H, et al. Genetic interaction of PICALM and APOE is associated with brain atrophy and cognitive impairment in Alzheimer's disease. Alzheimer's \& dementia : the journal of the Alzheimer's Association 2014; 10 (5 Suppl): S269-76.

Naj AC, Jun G, Beecham GW, Wang LS, Vardarajan BN, Buros J, et al. Common variants at MS4A4/MS4A6E, CD2AP, CD33 and EPHA1 are associated with late-onset Alzheimer's disease. Nature genetics 2011; 43(5): 436-41.

Neumann M, Sampathu DM, Kwong LK, Truax AC, Micsenyi MC, Chou TT, et al. Ubiquitinated TDP-43 in frontotemporal lobar degeneration and amyotrophic lateral sclerosis. Science (New York, NY 2006; 314(5796): 130-3.

Nishikawa T, Takahashi T, Nakamori M, Yamazaki Y, Kurashige T, Nagano Y, et al. Phosphatidylinositol-4,5bisphosphate is enriched in granulovacuolar degeneration bodies and neurofibrillary tangles. Neuropathology and applied neurobiology 2014; 40(4): 489-501.

Nixon RA. Endosome function and dysfunction in Alzheimer's disease and other neurodegenerative diseases. Neurobiology of aging 2005; 26(3): 373-82.

Nixon RA. The role of autophagy in neurodegenerative disease. Nature medicine 2013; 19(8): 983-97.

Nixon RA, Yang DS. Autophagy failure in Alzheimer's disease--locating the primary defect. Neurobiology of disease 2011; 43(1): 38-45.

Pickford F, Masliah E, Britschgi M, Lucin K, Narasimhan R, Jaeger PA, et al. The autophagy-related protein beclin 1 shows reduced expression in early Alzheimer disease and regulates amyloid beta accumulation in mice. The Journal of clinical investigation 2008; 118(6): 2190-9.

Rohn TT, Catlin LW, Coonse KG, Habig JW. Identification of an amino-terminal fragment of apolipoprotein E4 that localizes to neurofibrillary tangles of the Alzheimer's disease brain. Brain research 2012; 1475: 106-15. Rohn TT, Day RJ, Catlin LW, Brown RJ, Rajic AJ, Poon WW. Immunolocalization of an Amino-Terminal Fragment of Apolipoprotein E in the Pick's Disease Brain. PloS one 2013; 8(12): e80180.

Rudinskiy N, Grishchuk Y, Vaslin A, Puyal J, Delacourte A, Hirling H, et al. Calpain hydrolysis of alpha- and beta2-adaptins decreases clathrin-dependent endocytosis and may promote neurodegeneration. The Journal of biological chemistry 2009; 284(18): 12447-58.

Saha AR, Ninkina NN, Hanger DP, Anderton BH, Davies AM, Buchman VL. Induction of neuronal death by alpha-synuclein. The European journal of neuroscience 2000; 12(8): 3073-7.

Saito K, Elce JS, Hamos JE, Nixon RA. Widespread activation of calcium-activated neutral proteinase (calpain) in the brain in Alzheimer disease: a potential molecular basis for neuronal degeneration. Proceedings of the National Academy of Sciences of the United States of America 1993; 90(7): 2628-32.

Sanders DW, Kaufman SK, DeVos SL, Sharma AM, Mirbaha H, Li A, et al. Distinct tau prion strains propagate in cells and mice and define different tauopathies. Neuron 2014; 82(6): 1271-88.

Sergeant N, Wattez A, Delacourte A. Neurofibrillary degeneration in progressive supranuclear palsy and corticobasal degeneration: tau pathologies with exclusively "exon 10" isoforms. Journal of neurochemistry 1999; 72(3): 1243-9.

Seshadri S, Fitzpatrick AL, Ikram MA, DeStefano AL, Gudnason V, Boada M, et al. Genome-wide analysis of genetic loci associated with Alzheimer disease. Jama 2010; 303(18): 1832-40.

Spillantini MG, Crowther RA, Kamphorst W, Heutink P, van Swieten JC. Tau pathology in two Dutch families with mutations in the microtubule-binding region of tau. The American journal of pathology 1998; 153(5): 135963.

Spillantini MG, Schmidt ML, Lee VM, Trojanowski JQ, Jakes R, Goedert M. Alpha-synuclein in Lewy bodies. Nature 1997; 388(6645): 839-40. 
Thal DR, Rub U, Orantes M, Braak H. Phases of A beta-deposition in the human brain and its relevance for the development of AD. Neurology 2002; 58(12): 1791-800.

Tian Y, Chang JC, Fan EY, Flajolet M, Greengard P. Adaptor complex AP2/PICALM, through interaction with LC3, targets Alzheimer's APP-CTF for terminal degradation via autophagy. Proceedings of the National Academy of Sciences of the United States of America 2013; 110(42): 17071-6.

Tian Y, Chang JC, Greengard P, Flajolet M. The convergence of endosomal and autophagosomal pathways: implications for APP-CTF degradation. Autophagy 2014; 10(4): 694-6.

Tracz E, Dickson DW, Hainfeld JF, Ksiezak-Reding H. Paired helical filaments in corticobasal degeneration: the fine fibrillary structure with NanoVan. Brain research 1997; 773(1-2): 33-44.

Wang Y, Mandelkow E. Tau in physiology and pathology. Nat Rev Neurosci 2016; 17(1): 22-35.

Uchihara T. Pretangles and neurofibrillary changes: similarities and differences between AD and CBD based on molecular and morphological evolution. Neuropathology 2014 ; 34(6):571-7.

Yao PJ. Synaptic frailty and clathrin-mediated synaptic vesicle trafficking in Alzheimer's disease. Trends in neurosciences 2004; 27(1): 24-9.

Yoshida M. Astrocytic inclusions in progressive supranuclear palsy and corticobasal degeneration.

Neuropathology : official journal of the Japanese Society of Neuropathology 2014; 34(6): 555-70.

Zhao Z, Sagare AP, Ma Q, Halliday MR, Kong P, Kisler K, et al. Central role for PICALM in amyloid-beta blood-brain barrier transcytosis and clearance. Nature neuroscience 2015; 18(7): 978-87.

Zhou Y, Hayashi I, Wong J, Tugusheva K, Renger JJ, Zerbinatti C. Intracellular clusterin interacts with brain isoforms of the bridging integrator 1 and with the microtubule-associated protein Tau in Alzheimer's disease. PloS one 2014; 9(7): e103187. 
Figure 1
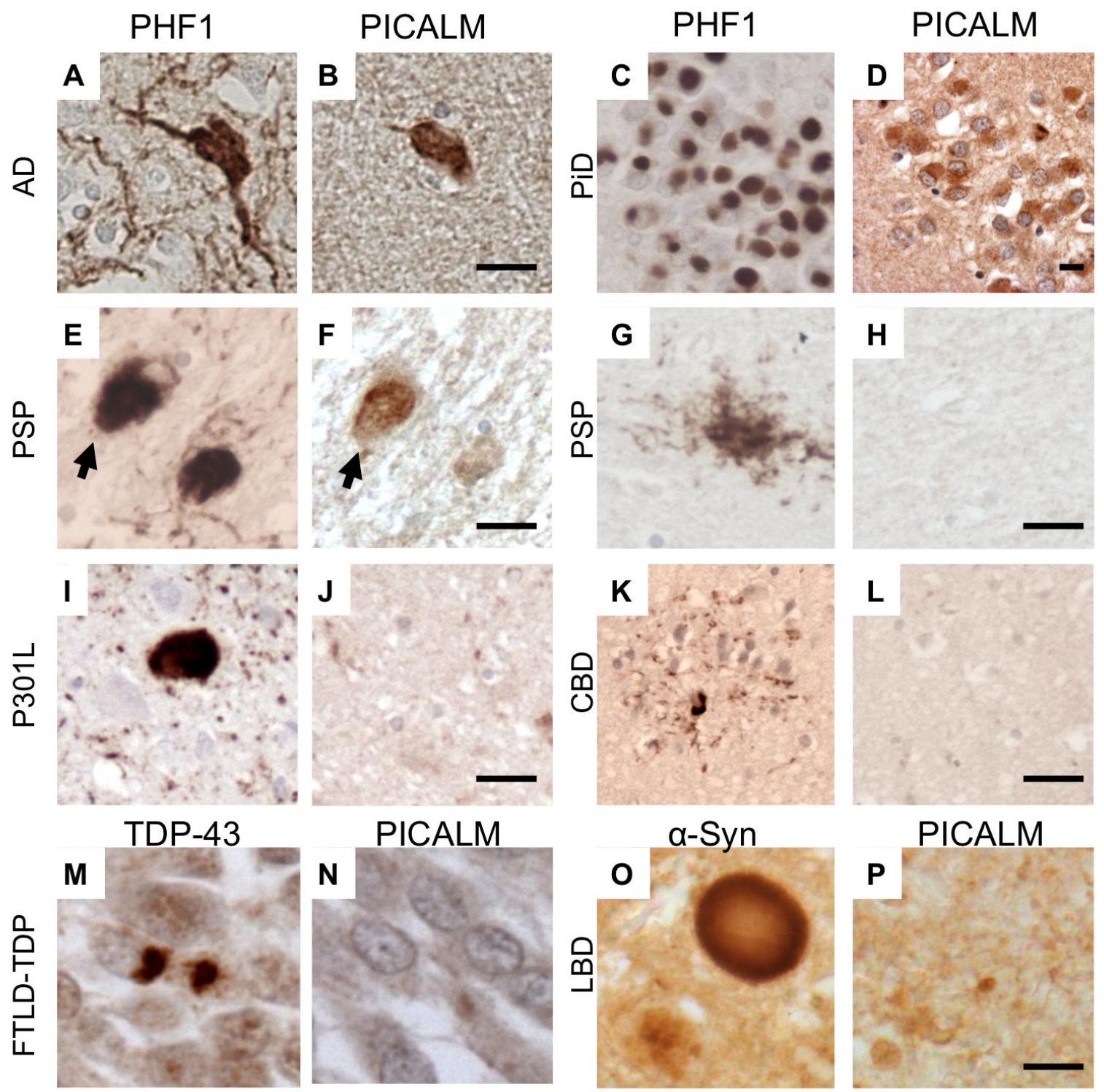


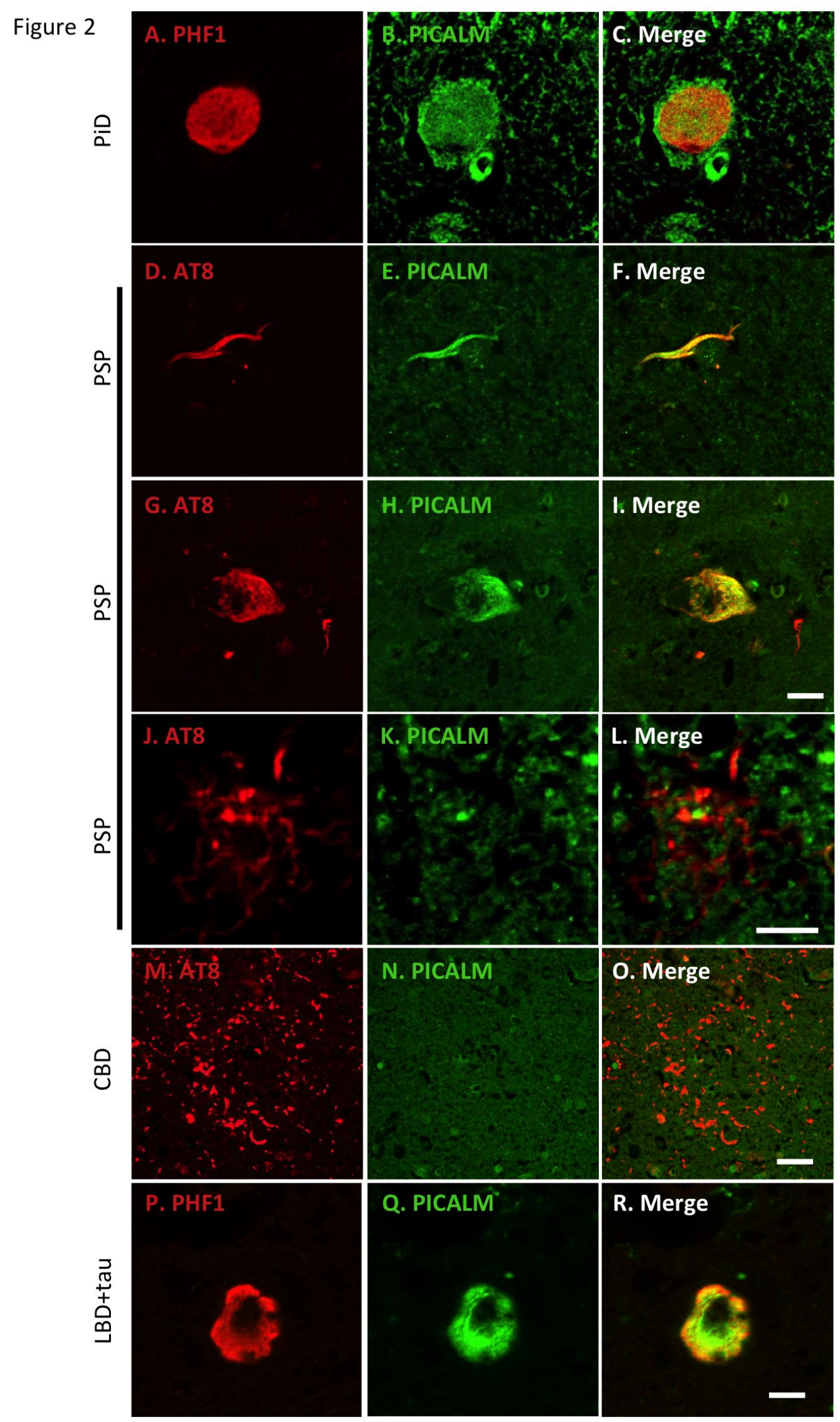


Figure 3
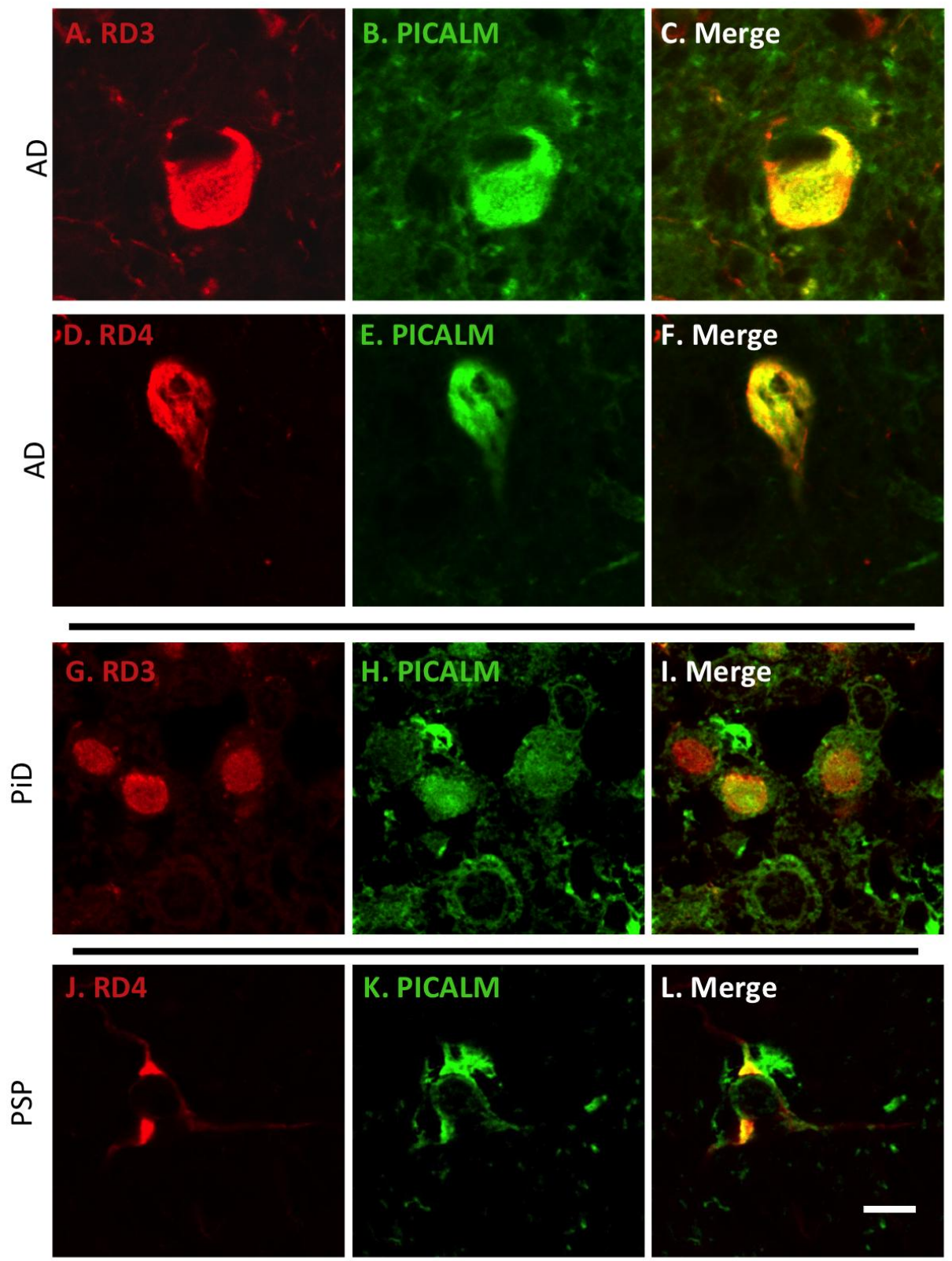
Figure 4
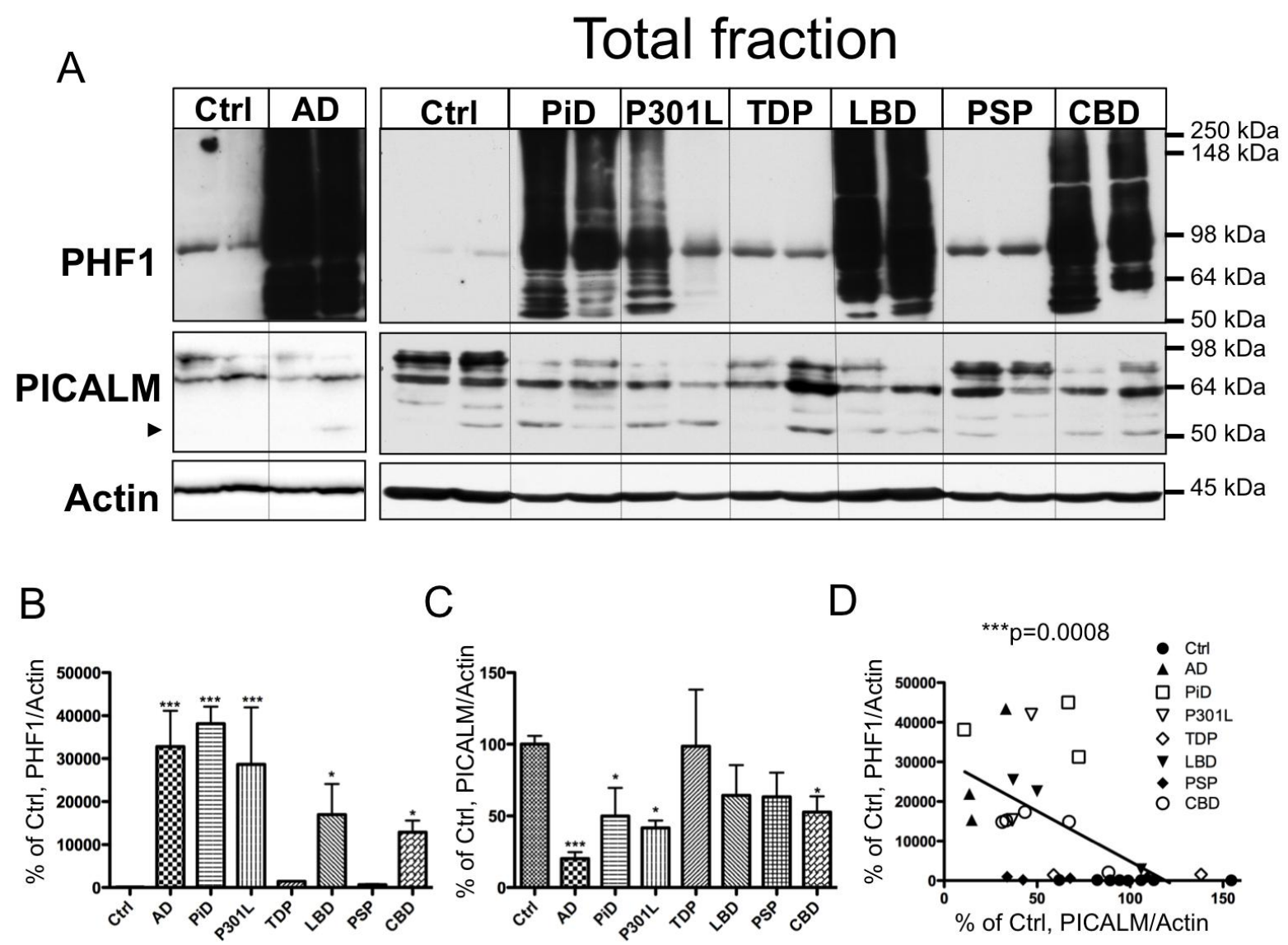
Figure 5

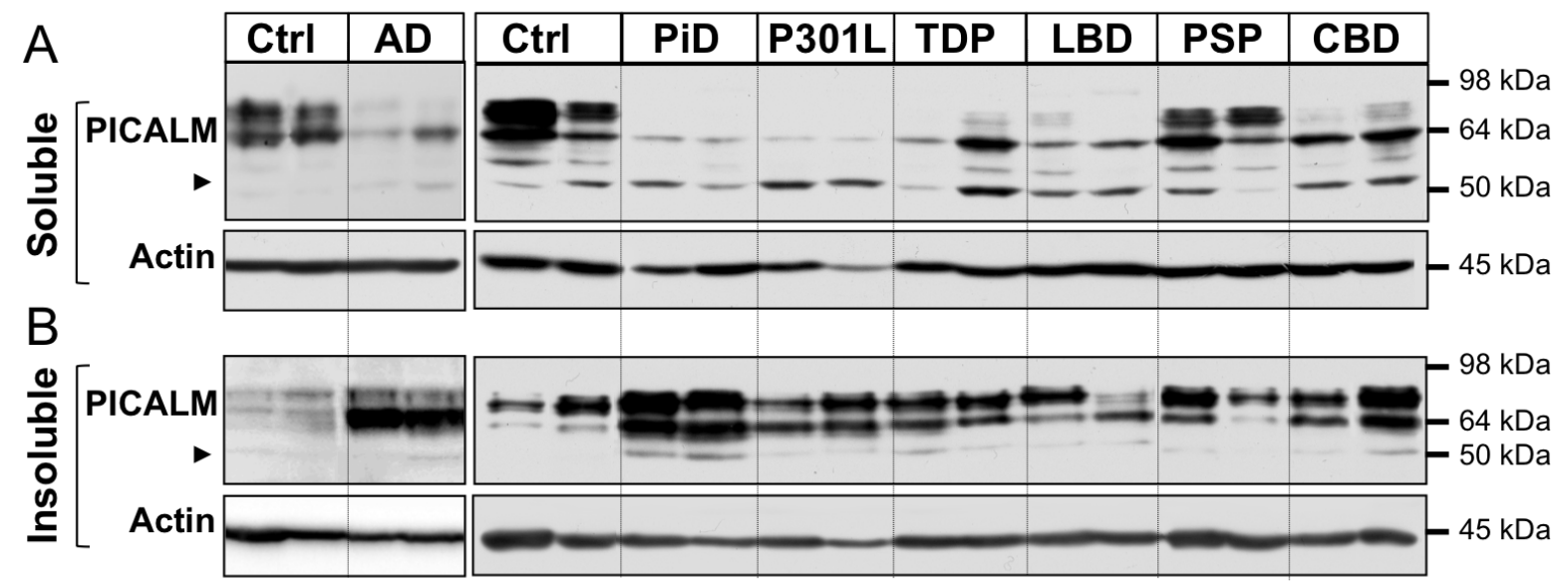

C

Soluble

D Insoluble
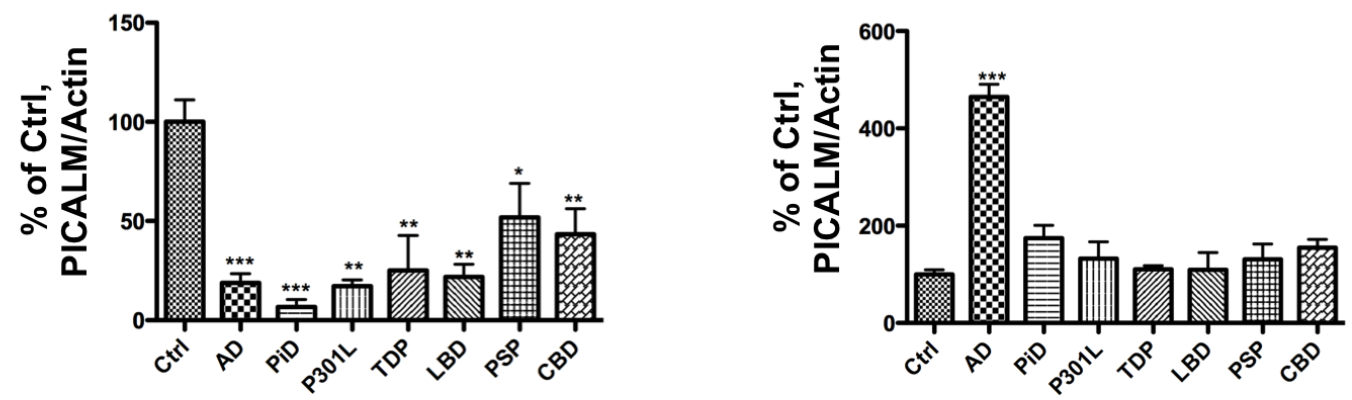

E

F
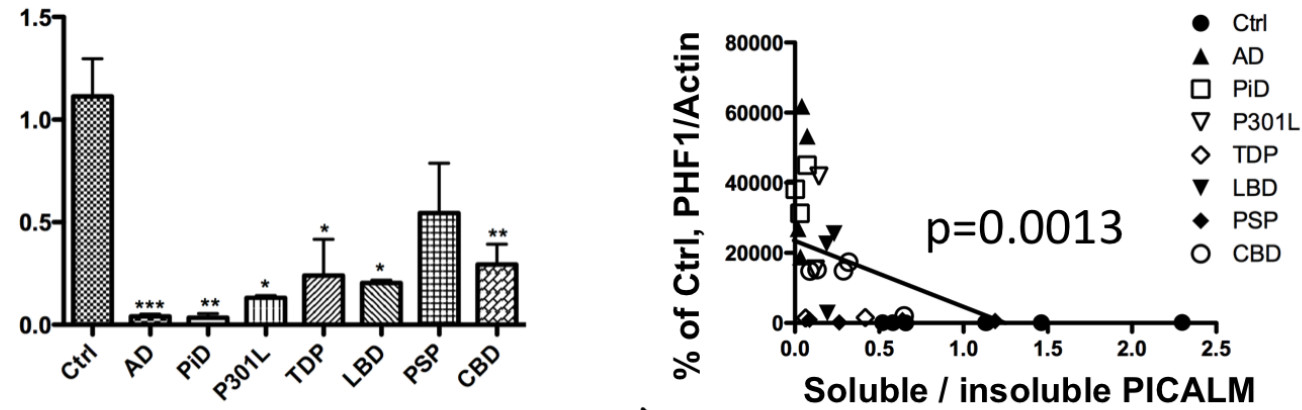
Figure 6

A

Total fraction

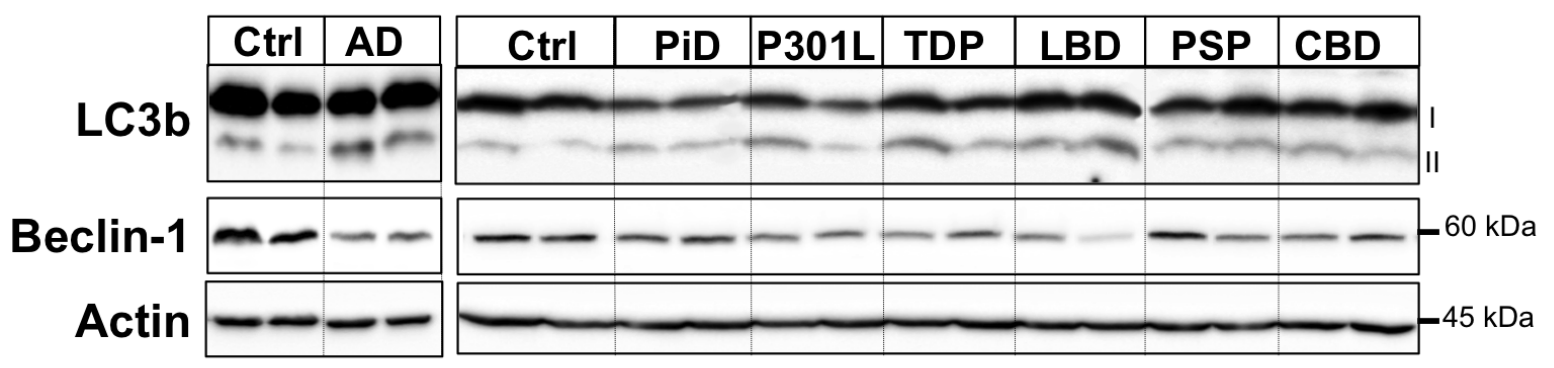

B

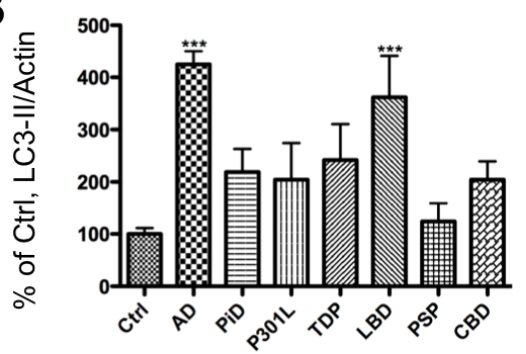

D

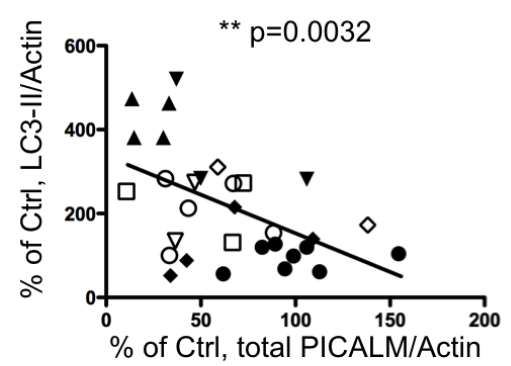

F

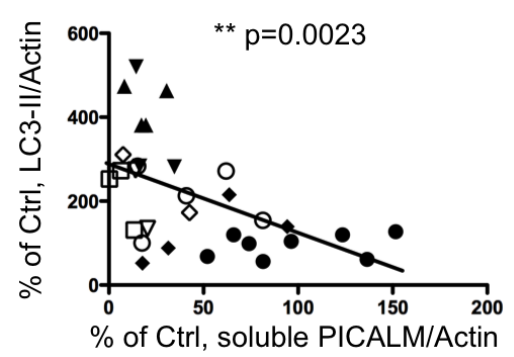

C

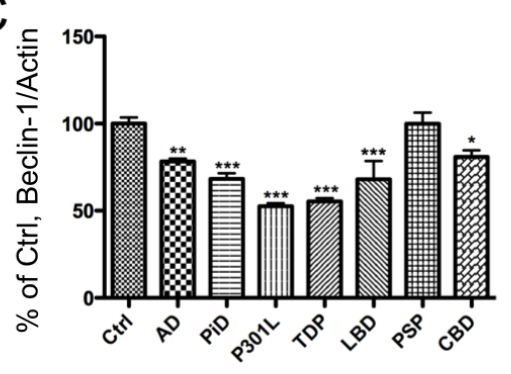

$E$

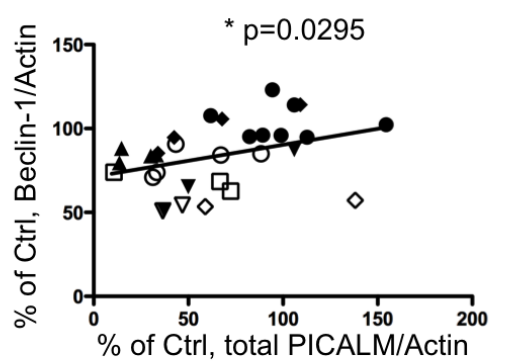

G

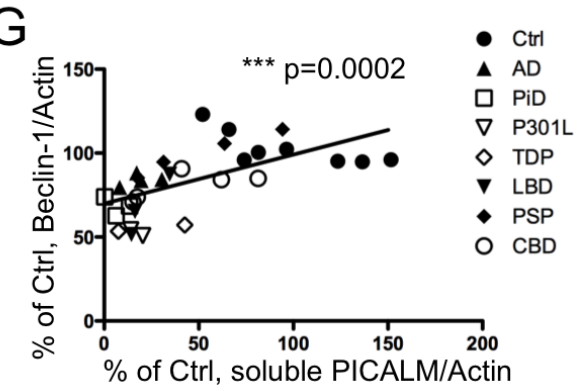


Table 1

\begin{tabular}{|c|c|c|c|c|c|c|c|}
\hline $\begin{array}{l}\text { Case } \\
\#\end{array}$ & Clinical diagnosis & Braak stage & $\begin{array}{l}\text { Thal stage } \\
\text { Plaque score }\end{array}$ & $\begin{array}{l}\text { PMD } \\
\text { (hours) }\end{array}$ & $\begin{array}{l}\text { Age } \\
\text { (years) }\end{array}$ & Sex & Analysis \\
\hline 1 & Control & 0 & 0 & 31 & 60 & $\mathrm{~F}$ & IHC, WB \\
\hline 2 & Control & II & 2 & 23 & 78 & M & IHC, WB \\
\hline 3 & Control & 0 & 0 & 6 & 69 & M & IHC, WB \\
\hline 4 & Control & III & 0 & 31 & 70 & M & IHC, WB \\
\hline 5 & Control & II & 0 & 26 & 66 & M & IHC, WB \\
\hline 6 & Control & IV & 2 & 29 & 77 & $\mathrm{~F}$ & $\mathrm{IHC}$ \\
\hline 7 & Control & 0 & 0 & 32 & 67 & $\mathrm{M}$ & IHC, WB \\
\hline 8 & Control & 0 & 0 & 5.5 & 58 & M & IHC, WB \\
\hline 9 & Control & I & 1 & NA & 92 & $\mathrm{~F}$ & IHC, WB \\
\hline 10 & $\mathrm{AD}$ & VI & 5 & 44 & 72 & M & IHC, WB \\
\hline 11 & $\mathrm{AD}$ & VI & 5 & 10 & 76 & M & IHC, WB \\
\hline 12 & $\mathrm{AD}$ & VI & 4 & 37 & 60 & M & IHC, WB \\
\hline 13 & $\mathrm{AD}$ & VI & 4 & 24 & 78 & $\mathrm{~F}$ & IHC, WB \\
\hline 14 & $\mathrm{AD}$ & VI & 5 & 45 & 73 & M & IHC \\
\hline 15 & Pick disease & NA & NA & 30 & 61 & M & IHC, WB \\
\hline 16 & Pick disease & NA & NA & 48 & 77 & M & IHC, WB \\
\hline 17 & Pick disease & NA & NA & NA & 67 & $\mathrm{~F}$ & IHC, WB \\
\hline 18 & P301L & NA & NA & 30 & 66 & M & IHC, WB \\
\hline 19 & P301L & NA & NA & 31 & 65 & $\mathrm{~F}$ & IHC, WB \\
\hline 20 & TDP (GRN) & 0 & 1 & 23 & 72 & M & IHC, WB \\
\hline 21 & TDP (C9) & II & 0 & NA & 49 & M & IHC, WB \\
\hline 22 & LBD & VI & NA & NA & 81 & M & IHC, WB \\
\hline 23 & LBD & VI & NA & 20.5 & 76 & M & IHC, WB \\
\hline 24 & LBD & VI & 5 & 28 & 83 & M & IHC, WB \\
\hline 25 & LBD & VI & 5 & 82 & 79 & M & IHC \\
\hline 26 & LBD & III & 3 & 24 & 61 & M & IHC \\
\hline 27 & LBD & I & 0 & 60 & 63 & $\mathrm{M}$ & IHC \\
\hline 28 & LBD & II & 0 & 25 & 70 & M & IHC \\
\hline 29 & PSP & NA & NA & 23 & 71 & M & IHC, WB \\
\hline 30 & PSP & NA & NA & NA & 71 & M & IHC, WB \\
\hline 31 & PSP & NA & NA & 14 & 71 & $\mathrm{~F}$ & IHC, WB \\
\hline 32 & PSP & NA & NA & 38 & 73 & M & IHC, WB \\
\hline 33 & $\mathrm{CBD}$ & VI & NA & 28 & 78 & $\mathrm{~F}$ & IHC, WB \\
\hline 34 & CBD & III & NA & NA & 77 & M & IHC, WB \\
\hline 35 & CBD & NA & NA & 23 & 74 & M & IHC, WB \\
\hline 36 & CBD & NA & NA & 33 & 81 & $\mathrm{~F}$ & IHC, WB \\
\hline 37 & CBD & NA & NA & 29 & 59 & M & IHC, WB \\
\hline
\end{tabular}


Ref: Ms. No.: NBD-16-203

"Level of PICALM, a key component of clathrin-mediated endocytosis, is correlated with levels of phosphotau and autophagy-related proteins and is associated with tau inclusions in AD, PSP and Pick disease" Ando et al

\section{Highlights}

- PICALM and phospho-tau are co-localized in NFT of AD, Pick disease and of PSP.

- Level of PICALM is decreased in tauopathies and inversely correlated with phosphotau.

- Level of PICALM is correlated with changes in autophagy markers in tauopathies.

- Reduction of soluble PICALM may play a key role in tauopathies by affecting autophagy.

(56 words) 